\title{
Error estimate of Taylor's frozen-in flow hypothesis in the spectral domain
}

\author{
Yasuhito Narita \\ Space Research Institute, Austrian Academy of Sciences, Schmiedlstr. 6, 8042 Graz, Austria \\ Correspondence to: Yasuhito Narita (yasuhito.narita@oeaw.ac.at)
}

Received: 27 December 2016 - Revised: 4 February 2017 - Accepted: 9 February 2017 - Published: 1 March 2017

\begin{abstract}
The quality of Taylor's frozen-in flow hypothesis can be measured by estimating the amount of the fluctuation energy mapped from the streamwise wavenumbers onto the Doppler-shifted frequencies in the spectral domain. For a random sweeping case with a Gaussian variation of the large-scale flow, the mapping quality is expressed by the error function which depends on the mean flow speed, the sweeping velocity, the frequency bin, and the frequency of interest. Both hydrodynamic and magnetohydrodynamic treatments are presented on the error estimate of Taylor's hypothesis with examples from the solar wind measurements.
\end{abstract}

Keywords. Interplanetary physics (plasma waves and turbulence) - meteorology and atmospheric dynamics (turbulence)

\section{Introduction}

Taylor's frozen-in flow hypothesis (Taylor, 1938) is widely used in the research of turbulent flows in various geophysical and space science applications. Taylor's hypothesis addresses the Doppler shift $\omega=k_{\mathrm{s}} U_{0}$ in the spectral domain, relating the measured frequency $\omega$ by the streamwise wavenumber $k_{\mathrm{S}}$ and the mean flow speed $U_{0}$. Using Taylor's hypothesis, one can map the fluctuation energy spectrum from the frequency domain onto the streamwise wavenumber domain and compare with the turbulence theories.

One may expect that Taylor's hypothesis is valid in a supersonic or super-Alfvénic flow because the intrinsic time variation such as sound waves or Alfvén waves is negligible. The presence of a large-scale flow variation, however, causes a Doppler broadening of the frequencies, and the quality of the mapping from the frequencies onto the wavenumbers becomes degraded. A good example and a bad example of Taylor's hypothesis are sketched in Fig. 1 based on the random sweeping model. A question arises naturally: "Is it possible to quantitatively estimate the mapping quality of Taylor's hypothesis?"

Here we develop a method to estimate the error of Taylor's hypothesis in the spectral domain using the random sweeping model (Kraichnan, 1964; Wilczek and Narita, 2012) assuming a Gaussian statistics of the large-scale flow variation. The existence of Doppler broadening is confirmed both theoretically for a random sweeping model (Wilczek and Narita, 2012) and observationally in interplanetary space (Narita, 2014). Starting with the hydrodynamic treatment for Taylor's hypothesis, we develop a magnetohydrodynamic approach to Taylor's hypothesis with an application to the solar wind measurements of Doppler shift and broadening.

The problem comes from the property of the wavenumberfrequency spectrum. According to the random sweeping model, the power-law index of the energy spectrum is the same between the frequency domain and the wavenumber domain even though there is significant frequency broadening by the sweeping velocity and Taylor's hypothesis is violated (Wilczek and Narita, 2012). The difference between a valid case and an invalid case of Taylor's hypothesis appears as a coefficient in the frequency spectrum such that the whole spectral curve is lifted, depending on the ratio of the sweeping velocity to the mean flow velocity (Wilczek et al., 2014). In other words, one cannot easily say whether Taylor's hypothesis is valid or not just by looking at the frequency spectrum.

It is true that the energy spectrum is measurable and is model-independent. On the other hand, the knowledge on the frequency broadening plays an important role in validating Taylor's hypothesis. The strength of the presented method is in its applicability to estimate the error of Taylor's hypothesis 

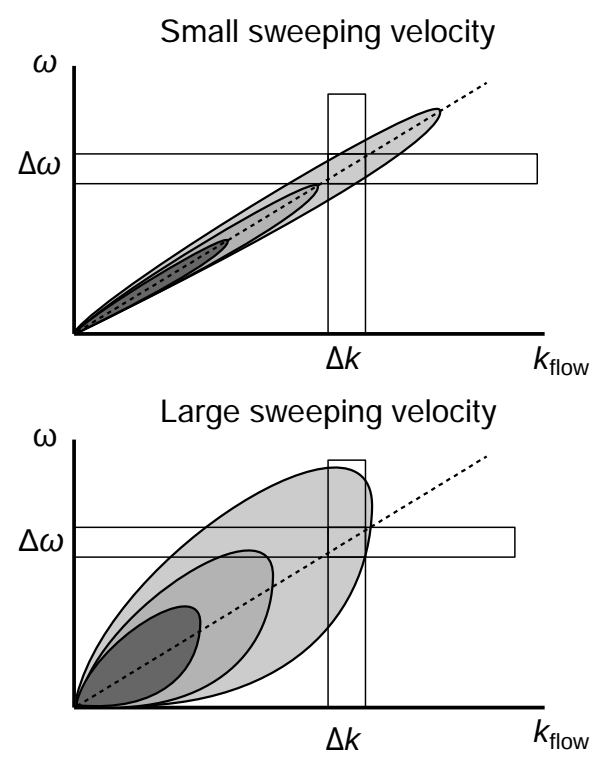

Figure 1. Sketches of wavenumber-frequency spectra for a good case of Taylor's hypothesis with a small sweeping velocity (upper panel) and a bad case with a large sweeping velocity (lower panel).

using only a small set of parameters without looking at the wavenumber-frequency spectrum.

\section{Hydrodynamic picture}

The random sweeping model is one representation of the turbulent fields and is based on the picture that the sweeping velocity is statistically of Gaussian nature, and is assumed to be not a function of the frequencies or wavenumbers. For a scale-dependent formulation of the large-scale fluctuating fields, the two-scale direct interaction approximation (Yoshizawa, 1984a, b, 1998) is a more valid approach.

The essence of the random sweeping model lies in introducing the frequency broadening function $F(\boldsymbol{k}, \omega)$. The energy spectrum is constructed in the wavenumberfrequency domain as $E(\boldsymbol{k}, \omega)=E(\boldsymbol{k}) F(\boldsymbol{k}, \omega)$ under the condition $\int_{-\infty}^{\infty} \mathrm{d} \omega F(\boldsymbol{k}, \omega)=1$ irrespective of the wavevector $\boldsymbol{k}$. An ideal situation of Taylor's hypothesis is restored for the Dirac delta function with the Doppler relation, $F(\boldsymbol{k}, \omega)=$ $\delta\left(\omega-\boldsymbol{k} \cdot \boldsymbol{U}_{0}\right)$.

In the random sweeping model, a Gaussian distribution is used as the broadening function. The first-order moment of the broadening function is the Doppler shift $k_{\mathrm{s}} U_{0}$. The second-order moment is the Doppler broadening $k_{\mathrm{s}} U_{\mathrm{rms}}$, where $U_{\text {rms }}$ is the root mean square of the flow speed. The Gaussian frequency broadening function is

$$
F(k, \omega)=\frac{1}{\sqrt{2 \pi k_{\mathrm{s}}^{2} U_{\mathrm{rms}}^{2}}} \exp \left[-\frac{\left(\omega-k_{\mathrm{s}} U_{0}\right)^{2}}{2 k_{\mathrm{s}}^{2} U_{\mathrm{rms}}^{2}}\right] .
$$

The measure of the validity of Taylor's hypothesis is defined as how much the fluctuation energy is transferred on the frequency bin between the Doppler-shifted frequency $k_{\mathrm{S}} U_{0}$ and $\omega_{1}=k_{\mathrm{s}} U_{0}+\Delta \omega$.

$$
I=2 \int_{k_{\mathrm{s}} U_{0}}^{k_{\mathrm{s}} U_{0}+\Delta \omega} \mathrm{d} \omega \frac{1}{\sqrt{2 \pi k_{\mathrm{s}}^{2} U_{\mathrm{rms}}^{2}}} \exp \left[-\frac{\left(\omega-k_{\mathrm{s}} U_{0}\right)^{2}}{2 k_{\mathrm{s}}^{2} U_{\mathrm{rms}}^{2}}\right]
$$

The factor 2 is introduced for a symmetric function with respect to the Doppler shift $k_{\mathrm{s}} U_{0}$. Taylor's hypothesis is valid or nearly valid if the measure $I$ is close to unity. The measure $I$ is equivalent to the error function by introducing a new variable $\tau$ and its derivative:

$$
\begin{aligned}
\tau & =\frac{1}{\sqrt{2}}\left(\frac{\omega}{k_{\mathrm{s}} U_{\mathrm{rms}}}-\frac{U_{0}}{U_{\mathrm{rms}}}\right), \\
\mathrm{d} \tau & =\frac{1}{\sqrt{2 k_{\mathrm{s}}^{2} U_{\mathrm{rms}}^{2}}} \mathrm{~d} \omega .
\end{aligned}
$$

The lower limit of the integration range is $\tau=0$. The upper limit is $\Delta \tau$ defined as

$$
\begin{aligned}
\Delta \tau & =\frac{1}{\sqrt{2}}\left(\frac{k_{\mathrm{s}} U_{0}+\Delta \omega}{k_{\mathrm{s}} U_{\mathrm{rms}}}-\frac{U_{0}}{U_{\mathrm{rms}}}\right), \\
& \simeq \frac{1}{\sqrt{2}} \frac{U_{0}}{U_{\mathrm{rms}}} \frac{\Delta \omega}{\omega},
\end{aligned}
$$

where the streamwise wavenumber is approximated by the mapped frequency, $k_{\mathrm{s}} \simeq \omega / U_{0}$. The measure $I$ is written then as

$$
\begin{aligned}
I & =\frac{2}{\sqrt{\pi}} \int_{0}^{\Delta \tau} \mathrm{d} \tau \exp \left[-\tau^{2}\right] \\
& =\operatorname{erf}(\Delta \tau) .
\end{aligned}
$$

Eqs. (6) and (8) are the quantitative error estimate of Taylor's hypothesis for the random sweeping model. Taylor's hypothesis is more valid for a larger value of $\Delta \tau$, that is,

1. The mean flow speed $U_{0}$ is high.

2. The sweeping velocity $U_{\mathrm{rms}}$ is small.

3. The frequency bin $\Delta \omega$ is larger.

4. The applied frequency $\omega$ is low.

Condition (1) and (2) are an equivalent statement to the Doppler relation including the error, $\omega=k_{\mathrm{S}}\left(U_{0}+\delta U\right)$. Condition (3) comes from the fact that there is more fluctuation energy in the integration range for a larger frequency bin, and prefers a shorter period of measurements, which is equivalent to the time domain picture of Taylor's hypothesis. Otherwise the fluctuating field evolves during the measurement. Condition (4) represents the nature of the Doppler broadening by the sweeping velocity, $\delta \omega \sim k_{\mathrm{s}} U_{\mathrm{rms}}$. The broadening is 
larger at higher wavenumbers and hence at higher frequencies. Conditions (3) and (4) impose a challenge in the measurement; one should use low frequencies but the measurement time length should be shorter.

For example, the mean flow velocity and the sweeping velocity are of comparable order for oceanic turbulence, $U_{0}=0.01 \mathrm{~m} \mathrm{~s}^{-1}$ and $U_{\mathrm{rms}}=0.01 \mathrm{~m} \mathrm{~s}^{-1}$ (Lien et al., 1998; Lien and D'Asaro, 2006). For a frequency resolution of $\Delta f=0.1 \mathrm{~Hz}$ (Lien and D'Asaro, 2006), the measure of Taylor's hypothesis accuracy is $I \simeq 7.97 \times 10^{-2}$ (about $8 \%$ ) and $I \simeq 7.98 \times 10^{-3}$ (about $0.8 \%$ ) at frequencies of $f=1 \mathrm{~Hz}$ and $f=10 \mathrm{~Hz}$, respectively. The mapping accuracy is low, as is expected from the fact that the sweeping velocity is as large as the mean flow velocity. Equation (8) using the error function and the variable $\Delta \tau$ provides a practical method to quantify the validity of Taylor's hypothesis in the spectral domain.

\section{Magnetohydrodynamic picture}

The hydrodynamic formulation using the sweeping velocity is applicable to magnetohydrodynamic (MHD) turbulence under several assumptions. Complications arise in MHD turbulence in two aspects: (1) the Doppler shift becomes split into dispersion relations; (2) the concept of the sweeping velocity needs to be separated from the root mean square of the flow velocity variation.

The wavenumber-frequency spectrum for MHD turbulence is

$$
\begin{aligned}
E(\boldsymbol{k}, \omega) & =\frac{E_{(+)}(\boldsymbol{k})}{\sqrt{2 \pi\left(\sigma_{(+)}\right)^{2}}} \exp \left[-\frac{\left(\omega-\boldsymbol{k} \cdot \boldsymbol{U}_{(+)}\right)^{2}}{2\left(\sigma_{(+)}\right)^{2}}\right] \\
& +\frac{E_{(-)}(\boldsymbol{k})}{\sqrt{2 \pi\left(\sigma_{(-)}\right)^{2}}} \exp \left[-\frac{\left(\omega-\boldsymbol{k} \cdot \boldsymbol{U}_{(-)}\right)^{2}}{2\left(\sigma_{(-)}\right)^{2}}\right] .
\end{aligned}
$$

The above spectrum is derived from the linearized MHD equations (see Appendix). The energy spectra need to be evaluated for the forward component $E_{(+)}(\boldsymbol{k})$ the backward component $E_{(-)}(\boldsymbol{k})$. The difference between the two spectra is related to the cross helicity. The symbols $\boldsymbol{U}_{(+)}=\boldsymbol{U}_{0}+$ $\boldsymbol{V}_{\mathrm{A}}$ and $\boldsymbol{U}_{(-)}=\boldsymbol{U}_{0}-\boldsymbol{V}_{\mathrm{A}}$ denote the Doppler-shifted Alfvén speed (forward for the plus sign and backward for the minus sign). The frequency broadenings are expressed as

$\sigma_{(+)}=\sqrt{\left|\boldsymbol{k} \cdot \delta \boldsymbol{U}_{(+)}\right|^{2}}$,

$\sigma_{(-)}=\sqrt{\left|\boldsymbol{k} \cdot \delta \boldsymbol{U}_{(-)}\right|^{2}}$,

where $\delta \boldsymbol{U}_{(+)}=\delta \boldsymbol{U}_{0}+\delta \boldsymbol{V}_{\mathrm{A}}$ and $\delta \boldsymbol{U}_{(-)}=\delta \boldsymbol{U}_{0}-\delta \boldsymbol{V}_{\mathrm{A}}$ denote the MHD sweeping velocities for the forward and backward components. $\delta \boldsymbol{U}_{0}$ and $\delta \boldsymbol{V}_{\mathrm{A}}$ represent the variation of the large-scale flow velocity and that of the large-scale wave field, respectively.

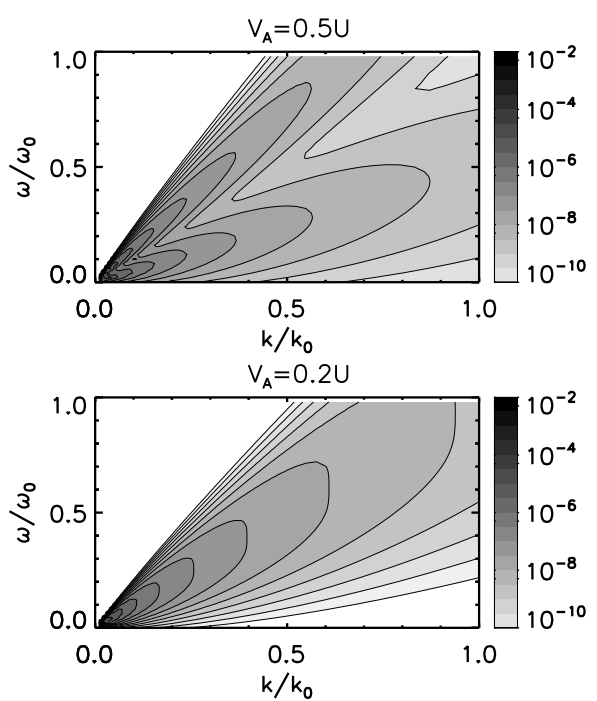

Figure 2. Wavenumber-frequency spectra with strong splitting (upper panel) and weak splitting (lower panel) for magnetohydrodynamic turbulence.

As is seen in Eq. (9), there are two spectral extensions in the wavevector-frequency domain. Due to the finite propagation speed, the extension splits into the Doppler-shifted, forward-propagating Alfvén waves and the Doppler-shifted, backward-propagating waves. Figure 2 displays two examples of the energy spectrum in the wavenumber-frequency domain. For simplicity, we take a special case in Fig. 2 in which the Alfvén waves (or the mean magnetic field) are in the direction of the mean flow, so that one direction is representative for discussion of the spatial structure. Depending on the ratio of the Alfvén speed to the flow speed, the energy spectrum may be clearly split (in the upper panel) or only weakly split (in the lower panel). In the latter case, the spectrum exhibits one extension along the Doppler relation, and the finite wave propagation effect makes the frequency broadening larger.

The direction of $\boldsymbol{V}_{\mathrm{A}}$ can be taken to be arbitrary (along the mean magnetic field or oblique). An important quantity to the MHD random sweeping model is the projection of the largescale wave propagation onto the mean flow direction (streamwise sense) by an angle of $\theta$. The streamwise wavenumberfrequency spectrum is

$$
\begin{gathered}
E\left(k_{\mathrm{s}}, \omega\right)=\frac{E_{(+)}\left(k_{\mathrm{S}}\right)}{\sqrt{2 \pi\left(\sigma_{(+)}\right)^{2}}} \exp \left[-\frac{\left(\omega-k_{\mathrm{S}}\left(U_{0}+V_{\mathrm{A}} \cos \theta\right)\right)^{2}}{2\left(\sigma_{(+)}\right)^{2}}\right] \\
+\frac{E_{(-)}\left(k_{\mathrm{S}}\right)}{\sqrt{2 \pi\left(\sigma_{(-)}\right)^{2}}} \exp \left[-\frac{\left(\omega-k_{\mathrm{S}}\left(U_{0}-V_{\mathrm{A}} \cos \theta\right)\right)^{2}}{2\left(\sigma_{(-)}\right)^{2}}\right] .
\end{gathered}
$$

The number of the free parameters in modeling the wavenumber-frequency spectrum is larger in MHD turbulence (Eq. 9) than in hydrodynamic model (see the broadening function in Eq. 1). The extensions are determined by the 
Doppler shift and the Alfvén speed, and the frequency broadening may be different between the forward and the backward wave components, $\sigma_{+}$and $\sigma_{-}$. Also, the wavevector dependence cannot be determined in the linearized treatment here. Since the propagation speeds of the Alfvén waves are anisotropic, it is natural to anticipate that the energy spectrum is anisotropic. Another complication comes from the fact that electromagnetic waves in plasmas become dispersive (variable phase speed) and dissipative (damping due to wave-particle resonances) on the small scales at the particle gyro-radius or smaller. In such a kinetic regime, individual particle motion needs to be considered, and magnetohydrodynamic treatment if no longer valid.

\section{Weak splitting approximation}

The MHD wavenumber-frequency spectra show two peak lines as a result of splitting of the Doppler shift into the forward-shifted Alfvén speed and the backward-shifted Alfvén speed (Fig. 2 upper panel). If the splitting is sufficiently weak, the spectral peaks follow the Doppler shift, while the sweeping velocity is renormalized using the Alfvén speed and the propagation angle. Observationally, the MHD random sweeping velocity is reported as being of the order of Alfvén speed, allowing deviation by a factor of about 2; see also Table 1 for two events of Cluster spacecraft measurements in the solar wind (discussed below). Figure 2 lower panel displays a weak splitting case.

In the case of zero cross helicity $\left(E_{(+)}=E_{(-)}\right)$, the symmetry between $\sigma_{(+)}=\sigma_{(-)}=\sigma$, and weak splitting, one may further simplify the wavenumber-frequency spectrum as follows. First, we approximate the frequency term as follows:

$$
\begin{aligned}
& \left(\omega-k_{\mathrm{S}}\left(U_{0} \pm V_{\mathrm{A}} \cos \theta\right)\right)^{2} \simeq\left(\omega-k_{\mathrm{S}} U_{0}\right)^{2} \\
& \quad \mp 2 \omega\left(\omega-k_{\mathrm{S}} U_{0}\right) k_{\mathrm{S}} V_{\mathrm{A}} \cos \theta .
\end{aligned}
$$

The second term on the right-hand side in Eq. (13) can be associated with the hyperbolic cosine function, and the spectrum is expressed as

$$
\begin{aligned}
E\left(k_{\mathrm{s}}, \omega\right)= & \frac{2}{\sqrt{2 \pi \sigma^{2}}} E\left(k_{\mathrm{s}}\right) \exp \left[-\frac{\left(\omega-k_{\mathrm{s}} U_{0}\right)^{2}}{2 \sigma^{2}}\right] \\
& \cosh \left[\frac{\left(\omega-k U_{0}\right) k_{\mathrm{s}} V_{\mathrm{A}} \cos \theta}{\sigma^{2}}\right] .
\end{aligned}
$$

It is interesting to observe that the exponential function and the hyperbolic cosine function compete against each other when the frequencies deviate from the Doppler shift. As a result, the hyperbolic cosine function contributes to additional frequency broadening.

A further approximation is possible by expanding the spectrum into a Taylor series and looking at the coefficient on the second-order term. We introduce temporary variables
Table 1. Parameters for the error estimate of Taylor's hypothesis for two events of Cluster spacecraft measurements of solar wind turbulence: event 1 from Narita et al. (2013) and event 2 from Narita (2014).

\begin{tabular}{lrr}
\hline Event & 1 & 2 \\
Date & 20 February 2002 & 12 February 2005 \\
Time & $19: 30-20: 00 \mathrm{UT}$ & $06: 30-08: 00 \mathrm{UT}$ \\
\hline$U_{0}$ & $444 \mathrm{~km} \mathrm{~s}^{-1}$ & $548 \mathrm{~km} \mathrm{~s}^{-1}$ \\
$U_{\text {swp }}$ & $195 \mathrm{~km} \mathrm{~s}^{-1}$ & $50 \mathrm{~km} \mathrm{~s}^{-1}$ \\
$V_{\mathrm{A}}$ & $85 \mathrm{~km} \mathrm{~s}^{-1}$ & $39 \mathrm{~km} \mathrm{~s}^{-1}$ \\
$U_{\mathrm{rms}}$ & $7 \mathrm{~km} \mathrm{~s}^{-1}$ & $20 \mathrm{~km} \mathrm{~s}^{-1}$ \\
$f_{\min }$ & $0.02 \mathrm{~Hz}$ & $0.001 \mathrm{~Hz}$ \\
$f_{\max }$ & $0.95 \mathrm{~Hz}$ & $0.2 \mathrm{~Hz}$ \\
$\Delta f$ & $0.02 \mathrm{~Hz}$ & $0.001 \mathrm{~Hz}$ \\
\hline
\end{tabular}

$x$ and $a$ to ease the calculation:

$x=\frac{\omega-k_{\mathrm{S}} U_{0}}{\sigma}$,

$a=\frac{k_{\mathrm{s}} V_{\mathrm{A}} \cos \theta}{\sigma}$.

The frequency dependence in the spectrum is then simplified to

$$
\begin{aligned}
\exp \left[-\frac{x^{2}}{2}\right] \cosh [a x] & =\left(1-\frac{x^{2}}{2}+\ldots\right)\left(1+\frac{(a x)^{2}}{2}+\ldots\right), \\
& =1-\frac{1-a^{2}}{2} x^{2}+\ldots
\end{aligned}
$$

The frequency broadening changes from $\sigma$ into $\sigma^{\prime}=$ $\sigma / \sqrt{1-a^{2}}$. The sweeping velocity can be regarded as a renormalized quantity and is to be measured by simulations or experiments.

A practical approach regarding Eq. (14) is to use the hydrodynamic wavenumber-frequency spectrum as a phenomenological model for MHD turbulence:

$E\left(k_{\mathrm{s}}, \omega\right)=\frac{E\left(k_{\mathrm{s}}\right)}{\sqrt{2 \pi k_{\mathrm{s}}^{2} U_{\mathrm{swp}}^{2}}} \exp \left[-\frac{\left(\omega-k_{\mathrm{s}} U_{0}\right)^{2}}{2 k_{\mathrm{s}}^{2} U_{\mathrm{swp}}^{2}}\right]$.

Here the frequency broadening is expressed by the renormalized sweeping velocity as $\left(\sigma^{\prime}\right)^{2}=k_{\mathrm{s}}^{2} U_{\mathrm{swp}}^{2}$. Equation (19) is an approximate form of the simplest expression of wavenumber-frequency spectrum for MHD turbulence in a super-Alfvénic flow.

Let us apply the error estimate to measurements of turbulent fluctuations in the solar wind. Under a typical condition, one may use a mean flow speed of about $400 \mathrm{~km} \mathrm{~s}^{-1}$ and a sweeping velocity of about $40 \mathrm{~km} \mathrm{~s}^{-1}$ (taken from the Alfvén speed), a frequency bin of about $\Delta \omega=2 \pi / T \simeq$ $1.04 \times 10^{-2} \mathrm{rad} \mathrm{s}^{-1}$, and a wave period of $10 \mathrm{~min}$ (in the inertial range), $\omega \simeq 6.28 \times 10^{-2} \mathrm{rad} \mathrm{s}^{-1}$ (a period of $100 \mathrm{~s}$ ). The parameter $\Delta \tau$ is evaluated as $\Delta \tau=\frac{1}{\sqrt{2}} \frac{10 V_{\mathrm{A}}}{V_{\mathrm{A}}} \frac{100}{600} \simeq 1.18$. The 


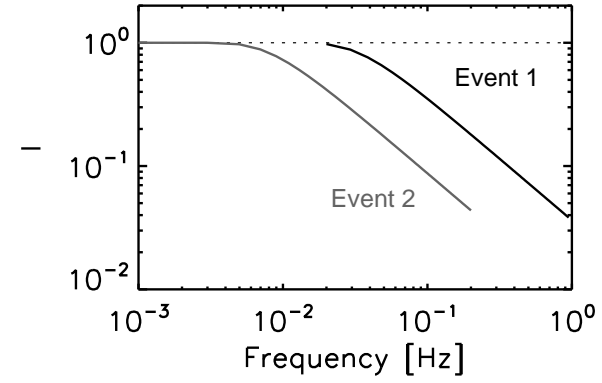

Figure 3. Measure of Taylor's hypothesis accuracy $I$ as a function of spacecraft-frame frequencies in the solar wind for event 1 (Narita et al., 2013) and event 2 (Narita, 2014).

measure of Taylor's hypothesis accuracy is $I=\operatorname{erf}(1.18) \simeq$ 0.90 . Therefore, $90 \%$ of the fluctuation energy is mapped from the wavenumber onto the frequency. For a $30 \mathrm{~min}$ measurement, the parameter is $\Delta \tau \simeq 0.39$ and the measure $I$ is $\operatorname{erf}(\Delta \tau) \simeq 0.42$. Only about $40 \%$ of the fluctuation energy comes from the mapping by the Doppler shift.

The profile of the measure $I$ is plotted as a function of spacecraft-frame frequencies for two cases of Cluster spacecraft measurements in the solar wind (Table 1 and Fig. 3). The mapping quality is high (the measure $I$ is close to unity) at lower frequencies around $0.01 \mathrm{~Hz}$ and below. The value of the measure $I$ falls as the error function $\operatorname{erf}(\Delta \tau)$ down to $10 \%$ and even below.

\section{Outlook}

It is instructive to note that the random sweeping model predicts that the one-dimensional frequency spectrum (integrated over the wavenumbers) appears as a power law irrespective of the sweeping velocity if the wavenumber spectrum (integrated over the frequencies) is a power law. Furthermore, the spectral index is the same both in the frequency domain and in the wavenumber domain (Wilczek and Narita, 2012). The effect of the sweeping velocity appears in the coefficient of the frequency spectrum. The sweeping velocity is the root mean square of the large-scale flow velocity variation in the hydrodynamic treatment. In MHD turbulence, in contrast, the situation is more complicated due to the splitting of the Doppler shift into the Alfvén waves.
In the hydrodynamic case, the random sweeping velocity can be regarded as the root-mean-square large-scale flow velocity variation. In the MHD case, the notion of the sweeping velocity is generalized into a renormalized quantity. Even though many parameters are involved in the formulation of the MHD wavenumber-frequency spectrum, the formulation with the single sweeping velocity provides a useful method to estimate the error of Taylor's hypothesis. A more theoretical formulation of the MHD sweeping velocity and the observational test is left for a future work.

The assumption of Gaussian frequency distribution breaks down if large-scale field variations show intermittency and phase coherence. Previous studies (Narita et al., 2013; Narita, 2014) use the method of fitting between the measured spectrum and the model spectrum to determine the sweeping velocity. A different approach would be to compute the moments of the energy spectrum by regarding the spectrum as a probability distribution function. That is, one can slice the wavenumber-frequency spectrum at a given value of the wavenumber and obtain the spectrum slice over the frequency domain. Then one measures the second-order moment of the sliced spectrum around the Doppler-shifted frequency. Such a method would work as a model-independent way.

In the ion kinetic domain (at scales of the order of 100 to $400 \mathrm{~km}$ in the solar wind), the electromagnetic waves become dispersive such as ion cyclotron waves, kinetic Alfvén waves and whistler waves. The dispersion relation is no longer linear between the wavenumbers and the frequencies. The kinetic extension of the error estimate for Taylor's hypothesis needs to include the dispersion effect.

It is true that one needs access to the wavenumberfrequency spectrum to fully validate Taylor's hypothesis and therefore multi-spacecraft missions from an observational point of view. But the applicability of the error estimate is not limited to multi-spacecraft missions. In particular, one can perform direct numerical simulations and study the error of Taylor's hypothesis systematically. The method in the paper can be used as a test for Taylor's hypothesis under different conditions of turbulent flow. 


\section{Appendix A: Solution of linearized MHD equations}

The random sweeping model can be extended to a magnetohydrodynamic treatment. We begin with a set of linearized magnetohydrodynamic equations:

$$
\begin{aligned}
& \frac{\partial \boldsymbol{u}}{\partial t}+\mathrm{i} \boldsymbol{k} \cdot\left(\boldsymbol{U}_{0}+\boldsymbol{\delta} \boldsymbol{U}\right) \boldsymbol{u}=\mathrm{i} \boldsymbol{k} \cdot\left(\boldsymbol{V}_{\mathrm{A}}+\boldsymbol{\delta} \boldsymbol{V}_{\mathrm{A}}\right) \boldsymbol{b}, \\
& \frac{\partial \boldsymbol{b}}{\partial t}+\mathrm{i} \boldsymbol{k} \cdot\left(\boldsymbol{U}_{0}+\boldsymbol{\delta} \boldsymbol{U}\right) \boldsymbol{b}=\mathrm{i} \boldsymbol{k} \cdot\left(\boldsymbol{V}_{\mathrm{A}}+\boldsymbol{\delta} \boldsymbol{V}_{\mathrm{A}}\right) \boldsymbol{u},
\end{aligned}
$$

where the Lorentz force is expressed into the magnetic tension force term. The symbol i denotes the imaginary number unit. The magnetic field $\boldsymbol{b}$ is given in the units of velocity as the mean magnetic field is transformed into the Alfvén speed. The magnetic field is assumed to consist of three components: the large-scale constant magnetic field (which is the Alfvén speed) $\boldsymbol{V}_{\mathrm{A}}$, the large-scale slowly varying magnetic field $\boldsymbol{\delta} \boldsymbol{V}_{\mathrm{A}}$, and the small-scale, turbulent magnetic field $\boldsymbol{b}$. The viscous term is neglected, and the total pressure (a sum of the thermal and the magnetic pressures) is assumed to be constant. The spatial gradient is replaced by the wavevector by the Fourier transform. One can see that the momentum equation in MHD has two self-coupling terms. One is self-coupling of the flow between the large-scale components and the small-scale ones, and the other is self-coupling of the magnetic field between the two scales. The second equation is the induction equation. The electric field is expressed by the flow velocity and the magnetic field on the assumption of the frozen-in magnetic field (i.e., the resistivity is neglected), and the magnetic field is again expressed in the velocity units. The coupling is between the flow velocity and the magnetic field in the induction equation.

We construct a state vector using the small-scale flow $\boldsymbol{u}$ and the small-scale magnetic field $\boldsymbol{b}$ as

$w=\left(\begin{array}{c}u \\ b\end{array}\right)$

The two linearized MHD equations (Eqs. A1 and A2) can be arranged into a matrix form as

$$
\left(\begin{array}{cc}
\partial_{t}+\mathrm{i} \boldsymbol{k} \cdot\left(\boldsymbol{U}_{0}+\delta \boldsymbol{U}\right) & -\mathrm{i} \boldsymbol{k} \cdot\left(\boldsymbol{V}_{\mathrm{A}}+\delta \boldsymbol{V}_{\mathrm{A}}\right) \\
-\mathrm{i} \boldsymbol{k} \cdot\left(\boldsymbol{V}_{\mathrm{A}}+\delta \boldsymbol{V}_{\mathrm{A}}\right) & \partial_{t}+\mathrm{i} \boldsymbol{k} \cdot\left(\boldsymbol{U}_{0}+\delta \boldsymbol{U}\right)
\end{array}\right)\left(\begin{array}{c}
\boldsymbol{u} \\
\boldsymbol{b}
\end{array}\right)=0 .
$$

The matrix $\mathbf{M}$ on the left-hand side in Eq. (A4) can be diagonalized into $\mathbf{M}^{\prime}$ with two eigenvalues $\lambda_{1}$ and $\lambda_{2}$ as

$$
\begin{aligned}
\mathbf{M}^{\prime}= & \left(\begin{array}{cc}
\lambda_{1} & 0 \\
0 & \lambda_{2}
\end{array}\right) \\
= & \operatorname{diag}\left(\partial_{t} \mathrm{i} \boldsymbol{k} \cdot\left[\left(\boldsymbol{U}_{0}+\delta \boldsymbol{U}\right)+\left(\boldsymbol{V}_{\mathrm{A}}+\delta \boldsymbol{V}_{\mathrm{A}}\right)\right],\right. \\
& \left.\partial_{t} \mathrm{i} \boldsymbol{k} \cdot\left[\left(\boldsymbol{U}_{0}+\delta \boldsymbol{U}\right)-\left(\boldsymbol{V}_{\mathrm{A}}+\delta \boldsymbol{V}_{\mathrm{A}}\right)\right]\right) .
\end{aligned}
$$

The associated eigenvectors $\boldsymbol{w}_{1}$ and $\boldsymbol{w}_{2}$ (not normalized) are

$w_{1}=\left(\begin{array}{c}u \\ -u\end{array}\right)$

for the first eigenvalue $\lambda_{1}$, and

$w_{2}=\left(\begin{array}{l}u \\ u\end{array}\right)$

for the second eigenvalue $\lambda_{2}$. The solution of the linearized MHD equations can therefore be constructed as follows:

$$
\begin{aligned}
\left(\begin{array}{c}
\boldsymbol{u}(\boldsymbol{k}, t) \\
\boldsymbol{b}(\boldsymbol{k}, t)
\end{array}\right) & =\left(\begin{array}{c}
\boldsymbol{u}_{+}(\boldsymbol{k}, 0) \\
\boldsymbol{b}_{+}(\boldsymbol{k}, 0)
\end{array}\right) e^{-\mathrm{i} \boldsymbol{k} \cdot \boldsymbol{U}_{(+)} t} \\
& +\left(\begin{array}{c}
\boldsymbol{u}_{-}(\boldsymbol{k}, 0) \\
\boldsymbol{b}_{-}(\boldsymbol{k}, 0)
\end{array}\right) e^{-\mathrm{i} \boldsymbol{k} \cdot \boldsymbol{U}_{(-)} t},
\end{aligned}
$$

where $\boldsymbol{U}_{(+)}=\boldsymbol{U}_{0}+\boldsymbol{V}_{\mathrm{A}}$ and $\boldsymbol{U}_{(-)}=\boldsymbol{U}_{0}-\boldsymbol{V}_{\mathrm{A}}$.

The solution can be formulated as a linear combination of Alfvén waves with the Doppler shift and broadening in two different directions forward and backward with respect to the flow. There are two contributions to the frequency broadening. One is the random sweeping by the large-scale flow variation (such as eddies) and the other is the largescale Alfvén waves. The first term on the right-hand side in Eq. (A9) represents the forward-propagating Alfvén waves with the Doppler shift, and the second term is the backwardpropagating Alfvén waves with the Doppler shift. Because of linearization, the two different Alfvén wave populations do not interact with each other.

The energy spectrum is determined from the covariance matrix $\left\langle\boldsymbol{w} \boldsymbol{w}^{\dagger}\right\rangle$ in the wavevector-temporal domain:

$$
\begin{aligned}
E(\boldsymbol{k}, t) & =E_{(+)}(\boldsymbol{k}) \exp \left[-\mathrm{i} \boldsymbol{k} \cdot \boldsymbol{U}_{+} t-\frac{\left(\sigma_{(+)}\right)^{2} t^{2}}{2}\right] \\
& +E_{(-)}(\boldsymbol{k}) \exp \left[-\mathrm{i} \boldsymbol{k} \cdot \boldsymbol{U}_{-} t-\frac{\left(\sigma_{(-)}\right)^{2} t^{2}}{2}\right] .
\end{aligned}
$$

Fourier transform from the time domain into the frequency domain gives the energy spectrum in the wavevectorfrequency domain (Eq. 9). 
Competing interests. The author declares that he has no conflict of interest.

Acknowledgements. This work is financially supported by the Austrian Space Applications Programme at the Austrian Research Promotion Agency, FFG ASAP-12 SOPHIE, Solar Orbiter wave observation program in the heliosphere under contract 853994.

The topical editor, E. Roussos, thanks one anonymous referee for help in evaluating this paper.

\section{References}

Lien, R.-C. and D'Asaro, E. A.: Measurement of turbulent kinetic energy dissipation rate with a Lagrangian float, J. Atmos. Ocean. Tech., 23, 964-976, doi:10.1175/JTECH1890.1, 2006.

Lien, R.-C., D'Asaro, E. A., and Dairiki, G. T.: Lagrangian frequency spectra of vertical velocity and vorticity in highReynolds-number oceanic turbulence, J. Fluid Mech., 362, 177198, doi:10.1017/S0022112098008787, 1998.

Kraichnan, R. H.: Kolmogorov's hypotheses and Eulerian turbulence theory, Phys. Fluids, 7, 1723-1734, doi:10.1063/1.2746572, 1964.

Narita, Y.: Four-dimensional energy spectrum for space-time structure of plasma turbulence, Nonlin. Processes Geophys., 21, 4147, doi:10.5194/npg-21-41-2014, 2014.
Narita, Y., Glassmeier, K.-H., Motschmann, U., and Wilczek, M.: Doppler shift and broadening in solar wind turbulence, Earth Planets Space, 65, e5-e8, doi:10.5047/eps.2012.12.002, 2013.

Taylor, G. I.: The spectrum of turbulence, P. R. Soc. Lond. A, 164, 476-490, doi:10.1098/rspa.1938.0032, 1938

Wilczek, M. and Narita, Y.: Wave-number frequency spectrum for turbulence from a random sweeping hypothesis with mean flow, Phys. Rev. E, 86, 066308, doi:10.1103/PhysRevE.86.066308, 2012.

Wilczek, M., Xu, H., and Narita, Y.: A note on Taylor's hypothesis under large-scale flow variation, Nonlin. Processes Geophys., 21, 645-649, doi:10.5194/npg-21-645-2014, 2014.

Yoshizawa, A.: Statistical analysis of the deviation of the Reynolds stress from its eddy-viscosity representation, Phys. Fluids, 27, 1377-1387, doi:10.1063/1.864780, 1984a.

Yoshizawa, A.: Statistical theory for the diffusion of a passive scalar in turbulent shear flows, Phys. Soc. Japan, 53, 1264-1276, doi:10.1143/JPSJ.53.1264, 1984b.

Yoshizawa, A.: Two-Scale Direct Interaction Approximation, Hydrodynamic and Magnetohydrodynamic Turbulent Flows, Fluid Mechanics and its Applications, Ser. 48, 173-253, Springer Verlag, Dortrecht, the Netherlands, doi:10.1007/978-94-017-18103 6, 1998 . 